\title{
A delivery system for olfactory stimuli
}

\author{
BENJAMIN R. PALMER, CON STOUGH, and JOHN PATTERSON \\ Swinburne University of Technology, Hawthorm, Victoria, Australia
}

\begin{abstract}
This paper describes the design of a new method for controlling and administering olfactory stimulinamely, the hood system. The hood system involves a stream of vaporized odor (at known concentrations) mixed with odorless air and pumped (at a constant flow rate) into an oxygen therapy hood. It is designed to be used with odorants in solution, such as essential oils, as the olfactory stimulus. The use of oxygen therapy hoods allows for the precise control of a constant concentration of odorized air over time, while allowing subjects to breathe normally. The hood system provides a natural administration of olfactory stimuli and the exact determination of the stimulus concentration. The use of this system will allow experimental conditions to be completely defined and results and replication studies to be accurately interpreted. The hood system is portable, cost effective, and constructed from readily available components. It is proposed that the hood system could be adopted to suit a wide range of olfactory research, particularly that in which the effects of chronic exposure to olfactory stimuli on cognition are examined.
\end{abstract}

Aromatherapy (the therapeutic use of scented oils) has long involved the claim that the odors of essential oils not only have a therapeutic effect on a variety of diseases and ailments, but also modify a wide variety of psychological states. It has been suggested that the odors of these oils not only can influence subjective psychological attributes, such as emotions and mood, but also can exert neurophysiological effects, such as sedation and stimulation of the brain (Davis, 1988). Over recent years, there has been a growing interest in scientifically validating these claims.

Recent research has suggested that olfactory stimuli modify memory, concentration, and attention (Baron, 1990; Ludvigson \& Rottman, 1989; Schab 1990; Warm, Dember, \& Parasuraman, 1991). However, there are many inconsistent results within the literature from this and other olfactory research. Several factors may have contributed to these inconsistent results. Lawless (1991) has suggested that the effects of odor exposure might be too subtle to produce replicable results. Another possible explanation is the disparate methodological procedures employed in these studies, particularly in the administration of the olfactory stimulus. Few devices for olfactory stimulus control are commercially available, and this has lead to the construction of many one-of-a-kind odor delivery systems, for which the effectiveness of the delivery and control of olfactory stimuli is unknown (Cain, ComettoMuniz, \& De Wijk, 1992).

Generally, these one-of-a-kind delivery systems have been of the dynamic type - that is, systems in which odoriferous vapor flows continuously, carried by a dilution gas, (typically, odorless air) toward the nose of the sub-

Correspondence concerning this article should be addressed to J. Patterson, School of Biophysical Sciences and Electrical Engineering, Swinburne University of Technology, P.O. Box 218, Hawthorn, VIC 3122, Australia (e-mail: jpatterson@swin.edu.au). ject. A number of researchers have constructed dynamictype odor delivery systems that use aroma disc player diffusers (e.g., Ludvigson \& Rottman, 1989; Schab, 1990). These diffusers vaporize specified amounts of olfactory stimuli over controlled time periods, typically into a testing room designed to minimize outside air disturbances. However, these testing rooms cannot precisely control for variables that influence vaporized odor concentrations, such as the flow rate of both recirculated and outside (fresh) air (Cain et al., 1992). Such systems only allow investigators to report the amount of olfactory stimulus vaporized over time into a specified volume. They do not control the specific concentration of odorized air to which each subject is exposed, nor do they allow the specific concentrations at which a stimulus has an effect to be defined. Other researchers have constructed constant flow olfactometers (Lorig \& Roberts, 1990), which also diffuse specified amounts of odorous substances over specified time periods. However, these systems have similar limitations. Ineffective delivery and control of olfactory stimuli may cause difficulty in defining the concentration at which olfactory stimuli influence cognition and may make it difficult for future research to adequately replicate previous experimental conditions.

Other researchers have adapted static type odor delivery systems, in which the odoriferous vapor is in semienclosed volumes. For example, Baron (1990) "sprayed" commercial air fresheners into an experimental room; Knasko (1992) placed perfume blotters around a testing room; Schiffman, Suggs, and Sattely-Miller (1995) and Schiffman, Sattely-Miller, Suggs, and Graham (1995) had subjects "spray" themselves with commercial colognes; Torii et al. (1988) had subjects smell filter paper impregnated with essential oils. The use of these odor delivery systems does not allow for adequate replication, since the amount and concentration of olfactory stimulus cannot be determined. 
Arguably, one of the better odor delivery systems to appear in olfactory research to date is that of Warm et al. (1991). This odor delivery system consisted of an air pump, a charcoal filter, a $35-\mathrm{ml}$ glass reservoir housed in a refrigerator that maintained olfactory stimuli at a constant temperature of $70^{\circ} \mathrm{F}$, and a modified home oxygen mask. This system allowed the odor concentration at the mask to be controlled by the flow of air from the pump and the approximate concentration of the olfactory stimulus that subjects received to be measured. However, problems also exist with the delivery of olfactory stimuli via oxygen masks. For example, breathing through oxygen masks can alter subjects' normal respiration, causing them to breathe more deeply, depending on the available air flow from the mask. In addition, wearing an oxygen mask can restrict vision and impair speech, thereby restricting the use of experimental paradigms that involve them, which is required by many cognitive tests of attention, concentration, and memory.

In a review of techniques previously used in olfactory research, Cain et al. (1992) argue that odor environmental chambers provide the greatest systematic ability to control and deliver olfactory stimuli. Cain et al. report that, with environmental chambers, air flow can be precisely controlled and monitored, while allowing the adequate mixing of any stimulus without creating disturbing air currents. In addition, such chambers are reported to offer the greatest freedom for odor sampling by humans, providing the most natural administration of olfactory stimuli. However, setting up environmental chambers with the necessary appliances, such as heating and cool- ing systems, as well as desiccant and humidifying devices, is very expensive and requires much space. As a result, much of the research in which the effects of olfactory stimuli on cognitive function are examined has been restricted to more primitive delivery systems.

In order to scientifically claim that olfactory stimuli modify cognition, there is a need to establish experimental conditions that are completely defined and replicable. Furthermore, in order for olfactory stimuli to be validly used in practical settings, there is an urgent need to define the type(s) and concentration(s) of odors that influence cognition. There is a need for future research to establish not only an economical olfactory delivery system that can accurately control and administer olfactory stimuli, but also one that can be utilized by different laboratories. The present paper outlines the development of a new system that meets such requirements.

\section{THE ODOR DELIVERY SYSTEM}

The design of this odor delivery system is of the $d y$ namic type. It consists of an air pump, an aromatizer, and an oxygen therapy hood (OTH), as illustrated in Figure 1.

The operation of the delivery system involves a stream of vaporized odor (at known concentrations) mixed with odorless air and pumped (at a constant flow rate) into an OTH. It is designed to be used with odorants in solution, such as essential oils, as the olfactory stimulus. The hood system creates a constant symmetrical odorized environment inside the hood, in which subjects breathe normally. Despite this system's "unnatural" appearance, the

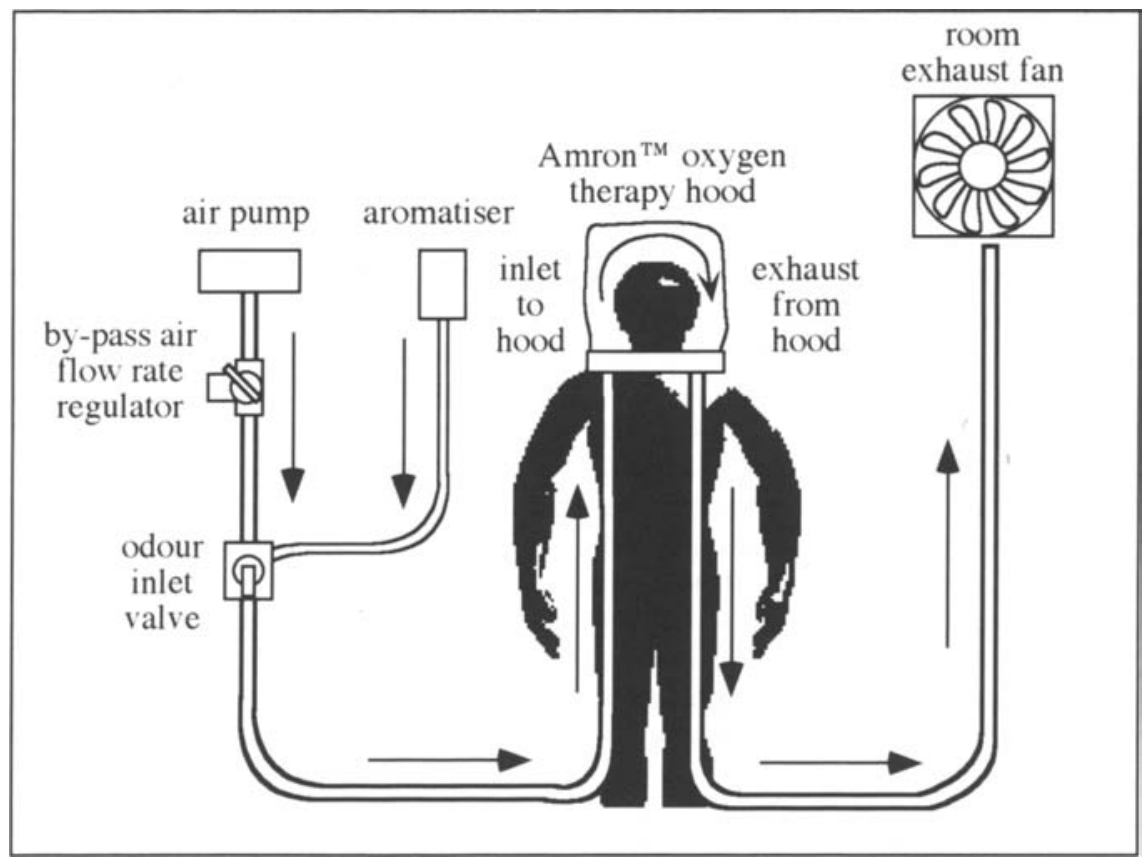

Figure 1. Diagram of the odor delivery system. 
odor delivery is "natural" in that the flow of stimulus to each nostril is determined by respiration, as air flow is not restricted or directed artificially within the hood.

\section{The Air Pump}

The purpose of the pump is to supply a flow of air to the OTH, which is connected to the pump via $20-\mathrm{mm}$ plastic tubing. First and foremost, the flow rate of the pump needs to be set at a level that ensures the safety of the subjects by preventing rebreathing of expired air inside the OTH. Continuous positive airway pressure (CPAP) also needs to be assessed, so that the pressure inside the OTH does not hinder normal breathing. These requirements are easily established by performing $\mathrm{CO}_{2}$ and CPAP analyses with a subject wearing the OTH. For the present study, the flow rate of a variable flow Sullivan CPAP pump (Model APD 2E, Medical Gases Ltd., Vic., Australia) was determined, using a MedGraphics Cardiopulmonary Exercise System (Model CPX-IOI, MedGraphics, Minneapolis, $\mathrm{MN}$ ), which contains an infrared $\mathrm{CO}_{2}$ gas analyzer. Placing a tube inside the OTH connected to a water column assessed CPAP. The flow rate of the pump was continually increased from $6 \mathrm{l} / \mathrm{min}$ until $\mathrm{CO}_{2}$ levels inside the hood were always below $0.05 \%$. A flow rate of $180 \mathrm{l} / \mathrm{min}$ was established to meet these criteria.

At this flow rate, subjects' normal breathing is not hindered either by rebreathing expired air or by positive air pressure. The second criterion of the airflow from the pump is to create the hood environment. The high flow rate of $180 \mathrm{l} / \mathrm{min}$ established previously was found to create a satisfactory hood environment. This flow rate provided a symmetrical and constant stream of odorized air (when being mixed with the olfactory stimulus) that remained constant over time and did not ramp up or ramp down with respiration (the inspired/expired volume is a small percentage of the hood volume). The high air flow rate also minimizes the adsorption of odor vapor, as well as condensation on the walls of the tubing and hood, keeping them from becoming a major source of contamination. This flow rate has also been observed to help cool subjects inside the hood, while continuously keeping the opticalquality viewing window free from fogging.

\section{Odor Vaporizer}

Olfactory stimuli are vaporized in this system by an Aromatiser Essential Oil Diffuser (model 101, Essential Therapeutics, Vic., Australia). This model aromatizer consists of a variable flow air pump and a nebulizer, which creates a stream of odorized air at vapor saturation for a given temperature. Odor concentration is determined by the airflow from the aromatizer pump; hence, a wide range of odor concentrations can be easily established by changing the pump flow rate and the mixing proportions. As an example, high and low odor concentrations of peppermint essential oil for two aromatizer pump settings are illustrated. Figure 2 shows the weight of the nebulizer with $1 \mathrm{ml}$ of peppermint and the weight after being vaporized for periods of $10,20,30$, and $40 \mathrm{~min}$.

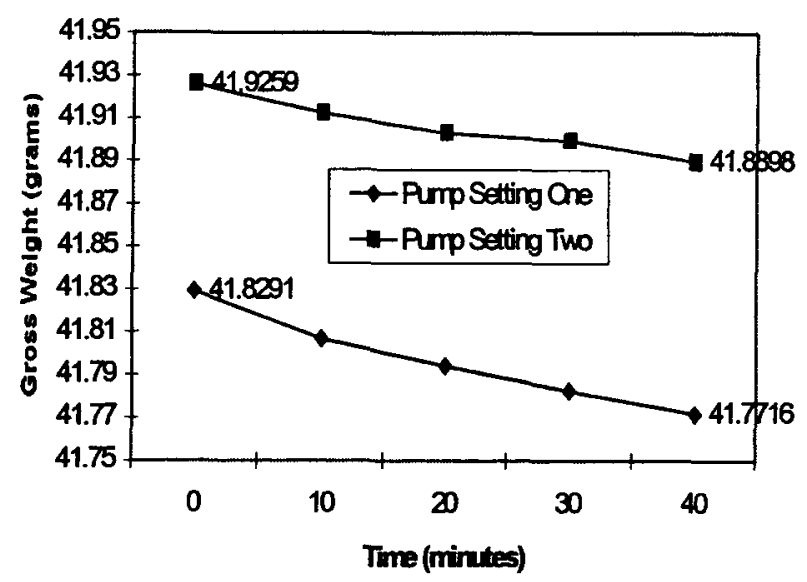

Figure 2. Weight of nebulizer and $1 \mathrm{ml}$ of oil being vaporized over time.

As is shown in Figure 2, $50.39 \mathrm{mg}$ of peppermint oil was vaporized over 40 min at pump setting one (PS1), and $36.1 \mathrm{mg}$ was vaporized at pump setting two (PS2). Figure 2 shows that the rate at which the aromatizer vaporizes peppermint oil is relatively constant over a period of $40 \mathrm{~min}$, both for PS1 and PS2. The amount of oil vaporized over 40 min established that, at PS 1 , the aromatizer vaporized peppermint oil at an average of $1.26 \mathrm{mg} /$ min, whereas at PS2, the aromatizer vaporized peppermint oil at an average of $0.90 \mathrm{mg} / \mathrm{min}$.

Independent referees rated the strength of the odor at the PS1 and PS2 settings. The scent of peppermint from the aromatizer at PS1 was, as expected, always rated as stronger than that at PS2 (while in the OTH), confirming PS1 and PS2 as high- and low-odor settings, respectively. Odor is delivered to the system by first opening the odor inlet valve, as is illustrated in Figure 1, and then switching the aromatizer pump on. To stop the olfactory stimulus (odor off), the aromatizer pump is switched off, and then the odor inlet valve is closed. This procedure is important, since it ensures that no leakage of odorized air occurs in the odor inlet line. With this procedure, the same subjects were consistently able to correctly identify the presence of the odor. The high flow rate from the air pump ensures that the odor quickly enters and leaves the hood environment when the odor inlet valve is opened and closed.

\section{The Oxygen Therapy Hoods}

Amron Oxygen Therapy Treatment Hoods (Amron International, California), shown in Figure 3, are used to contain the olfactory stimulus. The OTH comprises two main components, a neck ring and a clear soft-vinyl hood. The neck ring contains an O-ring seal for the vinyl hood attachment and has inlet and outlet locations designed to allow air to circulate throughout the hood with minimal noise. The inlet and outlet pipes are attached to the neck ring and are easily removed, providing an easy disconnect/reconnect procedure, should the subject need to 


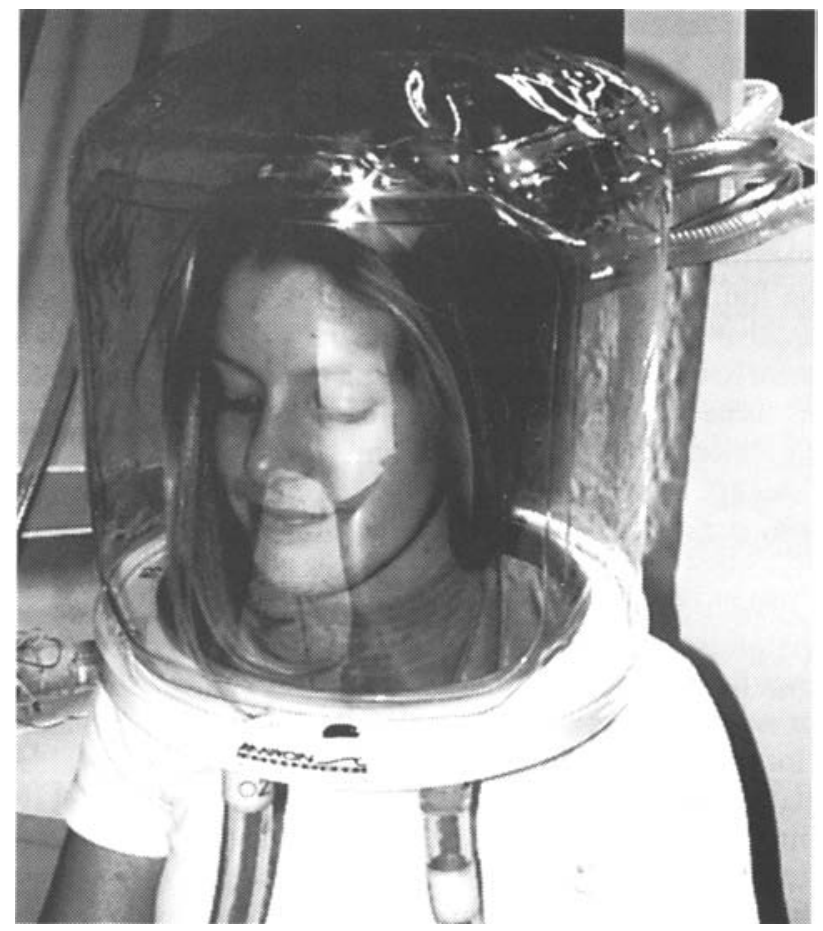

Figure 3. Amron oxygen therapy hood.

leave the experimental laboratory briefly. The hood attachment is also easily removed from the neck ring, should there be a need to, or should a subject wish to, stop the experiment or remove the hood quickly. The neck ring is placed over the head of the subject and has a replaceable surgical latex neck seal, which gently fits around the neck of the subject. The soft-vinyl hood has a large optical quality window, which allows visibility from the hood to be relatively unaltered.

The outlet from the hood is connected to $20-\mathrm{mm}$ tubing that carries the expired air and odor to an exhaust fan. This ensures that the exhaust from the hood is immediately removed and does not contaminate the room with odor. This also ensures that odor does not get drawn back into the air pump. Providing that the rest of the system is set up, it takes around $5 \mathrm{~min}$ to fit a subject in a hood and familiarize him or her with it. The hoods are easily cleaned in warm soapy water. The neck ring of the OTH has a replaceable surgical latex neck seal with various size trim lines. By measuring the neck size of a subject, a neck seal trimmed to size can be fitted, allowing for maximum comfort while wearing the hood. The unfamiliarity of the hood can cause some subjects to be apprehensive about wearing it. It is necessary to give subjects a demonstration of the equipment, allowing them to experience the flow of air through the hood and showing them how to remove it should they feel any need. Once subjects are acclimatized with the hood and realize how easily it can be removed, most feel at least comfortable, and some actually enjoy the novelty of wearing it.

\section{System Configuration}

The system should be installed in a temperaturecontrolled or constant temperature environment. This is important because essential oils are known to vaporize differently for given temperatures. The air pump and aromatizer are best kept out of the view of the subject by a subject-viewing shield, shown in Figure 4.

\section{SUMMARY}

Although the hood system cannot offer the freedom for odor sampling by humans that environmental chambers can, it does deliver constant odorized air over time. As with environmental chambers, the precise moment of olfactory stimulation cannot be specified. However, the hood system has major benefits for research involving chronic odor exposure, since the stimulus is constant over time and does not ramp-up and then ramp-down. Suitable methods for sampling the odor concentration are being investigated. The flow rate of fresh air is supplied and regulated by a variable flow air pump. Because the air going into the system is controlled and does not involve recirculated air, it is free from extraneous variables that can influence vaporized odor concentrations. Although the hood system does not provide a completely natural administration of olfactory stimuli, it does allow subjects to breathe normally, providing a somewhat natural administration of olfactory stimuli.

The hood itself is made of a clear soft-vinyl, which has a typical vinyl smell. However, when wearing the hood, 
the air flow (no aroma, just air from the air pump) minimizes the vinyl smell because of the high air flow rate necessary to maintain low ambient (in hood) $\mathrm{CO}_{2}$ concentration. Also, during testing involving odor and no-odor conditions, this plastic odor is always present during control (no-odor) and test (odor) conditions and is, therefore, counterbalanced. However, this background odor may form a synergistic relationship with some types of olfactory stimuli. It is important that there should be future research with the hood system to investigate the possible synergistic effect between this background odor and the olfactory stimulus. If this problem exists, it is suggested that the inside of the hood (except the viewing area) be coated with a teflon spray (i.e., Camie 2000 TFE coat), since this may reduce the possible effect without any substantive changes to the system. The large opticalquality window means that vision is essentially unrestricted by the hood. This allows for the administration of visual stimuli in olfactory or environmental research.

The air supply and exhaust locations on the neck ring of the OTH allow air to circulate easily throughout the hood without creating disturbing air currents and with minimal noise, while providing an odorized environment. As with environmental chambers, heating and cooling systems, as well as desiccant or humidifying devices, could be adopted according to the requirements of an experiment through the multipurpose port located in the neck ring. These accessories are, however, not necessary for the operation of this system, and therefore, the cost of the present design should prove considerably less than that associated with environmental chambers. Electrodes could be run through the multipurpose port, allowing EEG analysis to be carried out. The large soft-vinyl hood allows room for electrode caps to be worn while wearing the hood. Headphones could also be worn inside the hood, allowing the administration of auditory stimuli. This system could be modified to meet the requirements of most research in which the effects of olfactory stimulation on cognition are examined.

This system is compact and can be transported easily to different locations. Unlike research involving experimental chambers, the experimenters are in close contact with the subjects at all times, without exposure to odors. In addition, auxiliary equipment is not contaminated by ambient odors.

The multipurpose port on the neck ring of the hood allows for gas analysis via air sampling, in order to determine exact "air" environments inside the OTH. Further studies using this system will validate actual concentrations of different odors, which is necessary for every odor and every subject, given that different head volumes and respiratory rates will affect the actual dilution factor and, therefore, odor concentration, as with environmental chambers. The proposed system allows the experimental conditions to be completely defined and easily reproduced. Using gas chromatography analyses of samples of "hood air", the exact concentration of olfactory stimulus exposed to participants can be determined. The use of this system should allow future researchers to completely define experimental conditions, allowing for more accurate interpretations of results and replication studies.

The present paper outlines a feasibility study in which the human and technical aspects of the practicality of the hood system were evaluated. The hood delivery system provides solutions to some of the problems of odor delivery. This system may suit a wide range of chronic ex-

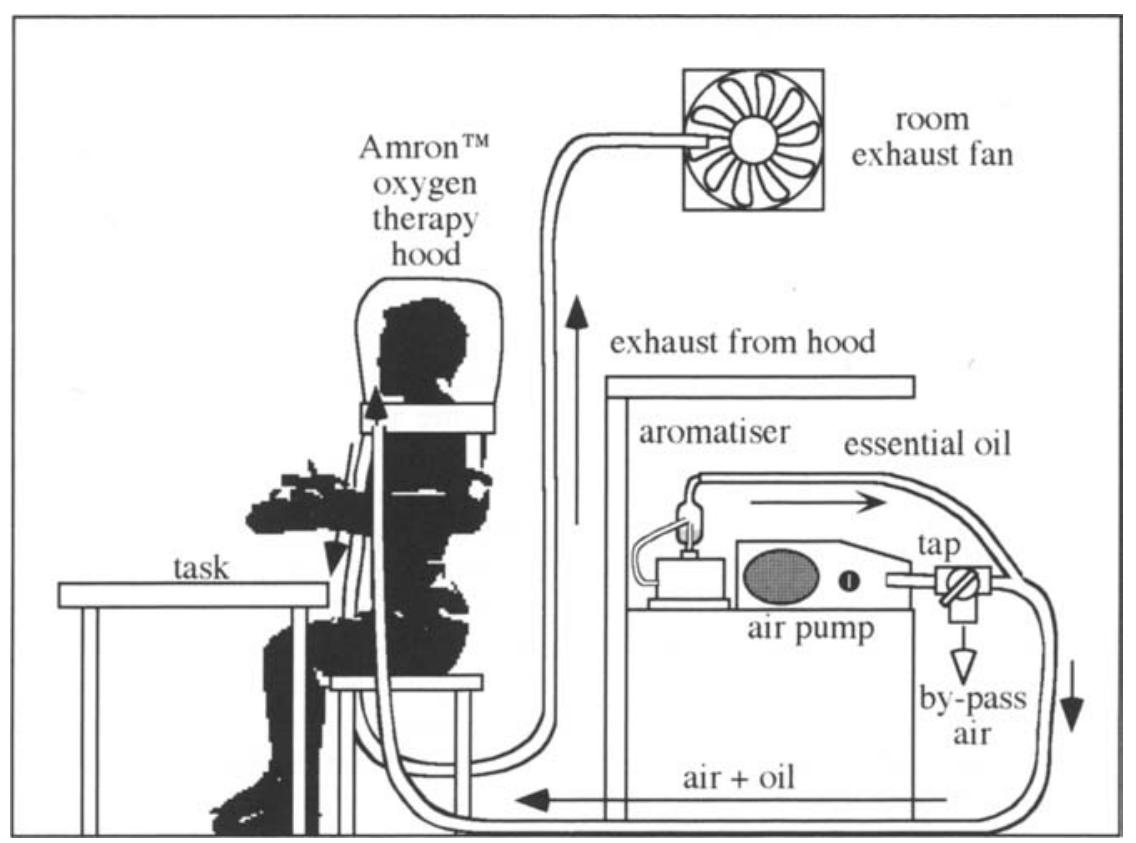

Figure 4. Experimental setting. 
posure studies similar to those that would utilize an environmental chamber or oxygen mask. Although the hood system may not be a solution to all delivery problems, it does provide a convenient, effective, and portable delivery device suitable for olfactory research involving the effects of olfactory stimulation over time.

\section{REFERENCES}

BARON, R. A. (1990). Environmentally induced positive affect: Its impact on self-efficacy, task performance, negotiation and conflict. Journal of Applied Social Psychology, 20, 368-384.

Cain, W.S., Cometro-Muniz, E., \& De WiJk, R. A. (1992). Techniques in the quantitative study of human olfaction. In M. J. Serby \& K. L. Chobor (Eds.), Science of olfaction (pp. 279-308). New York: SpringerVerlag.

DAvis, P. (1988). Aromatherapy: An A-Z. Saffron Waldon, U.K.: C. W. Daniel.

KNASKO, S. C. (1992). Ambient odor's effect on creativity, mood, and perceived health. Chemical Senses, 17, 27-35.

LAWLESS, H. (1991). Effects of odors on mood and behaviour: Aromatherapy and related effects. In D. G. Laing, R. L. Doty, \& W. Breipohl (Eds.), The human sense of smell (pp. 361-386). New York: SpringerVerlag.
LORIG, T. S., \& RoBerTs, M. (1990). The effects of odours on expectancy on the contingent negative variation. Chemical Senses, 15, 537-545.

Ludvigson, H. W., \& RotTMAN, T. R. (1989). Effects of ambient odors of lavender and cloves on cognition, memory, affect and mood. Chemical Senses, 14, 525-536.

ScHAB, F. R. (1990). Odors and the remembrance of things past. Journal of Experimental Psychology: Learning, Memory, \& Cognition, 16, 646-655.

Schiffman, S. S., Sattely-Miller, E. A., Suggs, M. S., \& Graham, B. G. (1995). The effect of pleasant odor and hormone status on mood of women at midlife. Brain Research Bulletin, 36, 31-37.

Schiffman, S. S., Suggs, M. S., \& Sattely-Miller, E. A. (1995). Effect of pleasant odors on mood of males at midlife: Comparison of African-American and European-American Men. Brain Research Bulletin, 36, 31-37.

Tori, S., Fukuda, H., Kanemoto, H., Miyanchi, R., Hamauza, Y., \& KAWASAKI, M. (1988). Contingent negative variation and the psychological effects of odor. In S. Van Toller \& G. Dodd (Eds.), Perfumery: The psychology and biology of fragrance (pp. 107-120). London: Chapman and Hall.

Warm, J. S., Dember, W. N., \& Parasuraman, R. (1991). Effects of olfactory stimulation on performance and stress in a visual sustained attention task. Journal of the Society of Cosmetic Chemists, 42, 199-210.

(Manuscript received August 26, 1998; revision accepted for publication June 23, 1999.) 